\title{
The Mechanism for Unimolecular Decomposition of RDX (1,3,5-Trinitro-1,3,5-triazine), an ab Initio Study
}

\author{
Debashis Chakraborty, Richard P. Muller, Siddharth Dasgupta, and William A. Goddard, III* \\ Materials and Process Simulation Center, Beckman Institute (139-74), Division of Chemistry and Chemical \\ Engineering, California Institute of Technology, Pasadena, California 91125
}

Received: October 18, 1999

\begin{abstract}
Gas phase hexahydro-1,3,5-trinitro-1,3,5-triazine (RDX) is a relatively stable molecule which releases a large amount of energy upon decomposition. Although gas-phase unimolecular decomposition experiments suggest at least two major pathways, there is no mechanistic understanding of the reactions involving RDX or other energetic molecules (such as HMX and TATB), used in applications ranging from automobile air bags to rocket propellants. For the unimolecular decomposition of RDX, we find three pathways: (i) concerted decomposition of the ring to form three $\mathrm{CH}_{2} \mathrm{NNO}_{2}(M=74)$ molecules, and (ii) homolytic cleavage of an $\mathrm{NN}$ bond to form $\mathrm{NO}_{2}(M=46)$ plus $\mathrm{RDR}(M=176)$, which subsequently decomposes to form various products. Experimental studies suggest that the concerted pathway is dominant while theoretical calculations have suggested that the homolytic pathway might require significantly less energy. We report here a third pathway: (iii) successive $H O N O$ elimination to form 3 HONO $(M=47)$ plus stable 1,3,5-triazine (TAZ) $(M$ $=81)$ with subsequent decomposition of HONO to $\mathrm{HO}(M=17)$ and $\mathrm{NO}(M=30)$ and at higher energies of TAZ into three HCN $(M=27)$. We examined all three pathways using first principles quantum mechanics (B3LYP, density functional theory), including the barriers for all low-lying products. We find: A threshold at $\sim 40 \mathrm{kcal} / \mathrm{mol}$ for which HONO elimination leads to TAZ plus $3 \mathrm{HONO}$, while $N N$ homolytic cleavage leads to RDR plus $\mathrm{NO}_{2}$, and the concerted pathway is not allowed; above $\sim 52 \mathrm{kcal} / \mathrm{mol}$ the TAZ of the HONO elimination pathway can decompose into $3 \mathrm{HCN}$ while the $\mathrm{HONO}$ can decompose into $\mathrm{HO}+\mathrm{NO}$; above $\sim 60 \mathrm{kcal} / \mathrm{mol}$ the concerted pathway opens to form $\mathrm{CH}_{2} \mathrm{NNO}_{2}$; at a threshold of $\sim 65 \mathrm{kcal} / \mathrm{mol}$ the RDR of the NN homolytic pathway can decompose into other products. These predictions are roughly consistent with previous experimental results and should be testable with new experiments. This should aid the development of a kinetic scheme to understand combustion and decomposition of solid-phase RDX and related energetic compounds (e.g., HMX).
\end{abstract}

\section{Introduction}

The cyclic nitramines hexahydro-1,3,5-trinitro-1,3,5-triazine (RDX) and octahydro-1,3,5,7- tetranitro-1,3,5,7-tetrazocine (HMX), are important energetic ingredients for various propellants and explosives since they release a large amount of energy in bulk decomposition. Thermal decomposition of these energetic materials has been observed to form very simple final product molecules, such as $\mathrm{HCN}, \mathrm{NO}, \mathrm{N}_{2} \mathrm{O}, \mathrm{NO}_{2}, \mathrm{CO}, \mathrm{CO}_{2}$, $\mathrm{H}_{2} \mathrm{O}, \mathrm{H}_{2} \mathrm{CO}$, etc. Understanding the underlying complex chemical processes is essential to obtain an improved model for combustion or detonation of these energetic materials.

A number of experimental studies ${ }^{1-20}$ have been directed toward elucidating the mechanistic details of the thermal decomposition of RDX, and various plausible reaction pathways have been proposed. Many experiments dealt with bulk phase materials, ${ }^{1-13}$ including decomposition in the condensed phase (solid or liquid) and the gas-phase flame structure near the burning RDX surface. The results reported have varied widely depending on the experimental conditions. To determine the initial steps of decomposition for these condensed phase studies, we focused on the gas-phase pyrolysis.

I.A. Experimental Studies. In an effort to understand the molecular level decomposition mechanism of RDX, Zhao,

\footnotetext{
* Corresponding author.
}

Hinsta, and Lee (ZHL), studied the infrared multiphoton dissociation (IRMPD) of RDX in a molecular beam using a time-of-flight velocity spectra (TOFVS). ${ }^{16}$ They detected mass fragments of $120,102,81,74,56,46,44,42,26-30$, and 1217 as the major products from laser photolysis (which includes mass fragments of both primary and secondary decomposition products). ZHL concluded that the dominant primary channel in the RDX decomposition is a concerted symmetric triple fission of RDX ring. Based on the reported heat of formation of $33.6 \mathrm{kcal} / \mathrm{mol}$ for methylenenitramine $(\mathrm{MN})$ by Melius and Binkley, ${ }^{21} \mathrm{ZHL}$ estimated the endothermicity to be $80 \mathrm{kcal} /$ mol for the concerted ring fission (which compares with the $\mathrm{N}-\mathrm{N}$ bond dissociation energy of $48 \mathrm{kcal} / \mathrm{mol}$ calculated by Melius and Binkley). ZHL estimated that the laser pulse in their experiments deposited a total internal energy of $80 \mathrm{kcal} / \mathrm{mol}$. Thus, they concluded "the concerted ring breaking is energetically accessible for a large fraction of the RDX in this experiment, even though the endoergicity derived from the heat of formation of MN given by Melius and Binkley seems unreasonably high".

On the other hand, more recent UV photolysis experiments ${ }^{18-20}$ observed $\mathrm{N}-\mathrm{NO}_{2}$ homolysis as the primary decomposition reaction.

Behrens and Bulusu ${ }^{2}$ using simultaneous thermogravimetry modulated beam mass spectrometry measurements, TOFVS 
analysis and isotopic crossover experiments suggested formation of oxy-s-triazine (OST) via the elimination of an HNO and two HONO molecules in the unimolecular decomposition of RDX in addition to other decomposition products. They concluded that OST is the only decomposition product from RDX that solely exhibits unimolecular decomposition kinetics. The rate of formation of OST has a first-order deuterium kinetic isotope effect which supports the HONO elimination as the ratecontrolling step.

Moreover, Capellos et al. ${ }^{17}$ based on photodissociation of RDX using $248 \mathrm{~nm}$ radiation ( $\mathrm{KrF}$ laser) considered that concerted HONO elimination through a five-member cyclic transition state (TS) is the most likely primary decomposition pathway.

In addition, formation of $\mathrm{N}_{2} \mathrm{O}_{4}$ was observed in the $\mathrm{CO}_{2}$ laser pyrolysis of RDX thin films. ${ }^{18}$ This result was interpreted mechanistically as $\mathrm{N}-\mathrm{NO}_{2}$ cleavage followed by dimerization of $\mathrm{NO}_{2}$ radical.

Recently, $\mathrm{CO}_{2}$ laser-induced pyrolysis of RDX, ${ }^{12}$ identified $47,54,56,70,81$, and 97 as the parent mass fragments below $m / e 100$ in addition to the usual small mass fragments $(18,27$, 30, 44, 46).

Summarizing, there is experimental evidence for both the concerted and $\mathrm{N}-\mathrm{N}$ homolysis dissociation pathways.

I.B. Theoretical Studies. The first qualitative theoretical analysis of the decomposition mechanism for RDX (Melius and Binkley ${ }^{21}$ ) used ab initio quantum mechanics at the MP4 level, corrected empirically (BAC-MP4). The conclusion was that the $\mathrm{N}-\mathrm{N}$ homolytic cleavage is the primary decomposition pathway. Assuming RDX has $\mathrm{N}-\mathrm{NO}_{2}$ bond dissociation energy similar to nitramine and methyl nitramine, they estimated a dissociation energy of $\sim 48 \mathrm{kcal} / \mathrm{mol}$ for RDX.

Harris and Lammertsma ${ }^{22}$ and Rice and Chabalowski ${ }^{23}$ reported detailed studies of the conformations and bond dissociation energies of RDX using ab initio (MP2) and density functional (DFT-B3LYP) methodologies. They suggested that several conformational minima for RDX exist within a small energy range of about $1 \mathrm{kcal} / \mathrm{mol}$. Furthermore, Harris and Lammertsma concluded that the $\mathrm{N}-\mathrm{NO}_{2}$ and $\mathrm{C}-\mathrm{H}$ bonds in RDX are unusually weak, indicating that the initiation of decomposition by $\mathrm{N}-\mathrm{N}$ bond cleavage and propagation of the decomposition by $\mathrm{H}$ atom transfer should be facile.

Sewell and Thompson ${ }^{24}$ used classical dynamics to study RDX decomposition. They estimated that the barrier height for the concerted ring breaking is $37 \mathrm{kcal} / \mathrm{mol}$ (based on available thermochemical information in the literature), confirming the experimental interpretation of ZHL. ${ }^{16}$ Later a similar study by Chambers and Thompson ${ }^{25}$ demonstrated that a concerted barrier height of $<40 \mathrm{kcal} / \mathrm{mol}$ is required to establish the branching ratio observed by $\mathrm{ZHL}$.

Habibollazadeh et al. ${ }^{26}$ carried out calculations at the semiempirical, ab initio, and DFT-LDA (local density approximation) level. They concluded that the barrier for the concerted fission is $72-75 \mathrm{kcal} / \mathrm{mol}$.

Recently, Wu and Fried ${ }^{27}$ carried out an extensive series of high-level DFT calculations (B3LYP, B3PW91, BPW91) of the potential energy profile for two dissociation channels: (I) $\mathrm{N}-\mathrm{NO}_{2}$ bond rupture and (II) concerted ring fission to three $\mathrm{MN}$ molecules. They found that path I is favored by $18.3 \mathrm{kcal} /$ mol over II, suggesting that $\mathrm{N}-\mathrm{NO}_{2}$ bond rupture is the dominant channel in gas-phase decomposition of RDX.

Summarizing, these theoretical studies have provided support for both the concerted and $\mathrm{N}-\mathrm{N}$ homolytic fission pathways, but somewhat favors the latter.
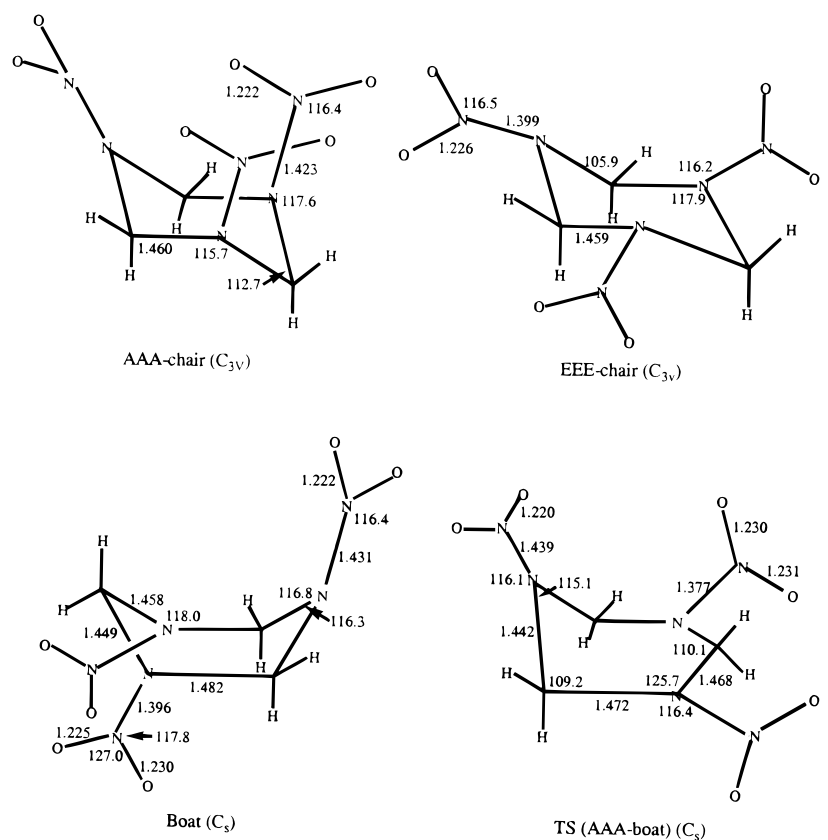

Figure 1. Optimized geometries of RDX in the AAA chair, EEE chair, and boat conformations and the corresponding boat to AAA chair transition state.

I.C. Current Work. Despite the large number of experimental and theoretical investigations, the mechanism of thermal decomposition of RDX is still uncertain. Experimentalists have observed numerous products in the thermal decomposition of RDX and have suggested two plausible qualitative schemes. ${ }^{13}$ Most theoretical calculations have been restricted to either conformational analysis of RDX or validation of the ZHL observations. Thus, they have considered just the two initial decomposition pathways, concerted ring fission versus $\mathrm{N}-\mathrm{N}$ bond cleavage. Consequently, we decided to carry out a detailed mechanistic study of thermal decomposition of RDX using ab initio DFT methodology (B3LYP).

Section II outlines the computational methods while Section III presents the calculational results. These are compared to experiment in Section IV, with the summary in Section V.

\section{Computational Methods}

The geometries of the reactants, products, intermediates, and transition states (TS) have been optimized at the B3LYP flavor of density functional theory. This includes the generalized gradient approximation (Becke 1988 nonlocal gradient correction), exact exchange using the Becke three parameter exchange functional, ${ }^{28}$ and the nonlocal correlation functional of Lee, Yang, and Parr. ${ }^{29}$ We used the modest 6-31G(d) basis set to be consistent with practical extensions of the same level of calculation to higher analogue such as HMX. This level of theory is expected to predict the energetics of large molecular systems such as RDX to a few $\mathrm{kcal} / \mathrm{mol}^{22,27}$

All stationary points have positively identified for local minima [number of imaginary frequencies $(\mathrm{NIMAG})=0$ ] and for TS $(\mathrm{NIMAG}=1)$. Vibrational frequencies were calculated at all stationary points to obtain zero-point energies (ZPE) and thermodynamic parameters. All calculations were carried out with Jaguar quantum chemistry program package. ${ }^{30}$

\section{Results}

Figure 1 shows the optimized geometry of RDX in two different conformations (chair and boat) and the corresponding 


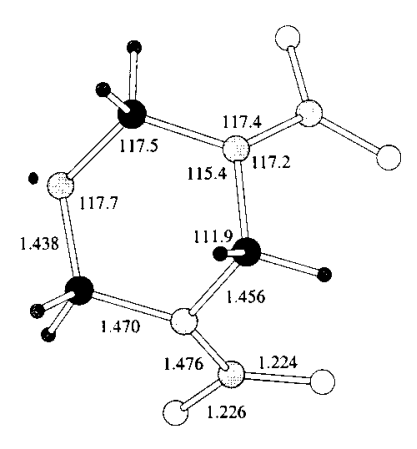

RDR

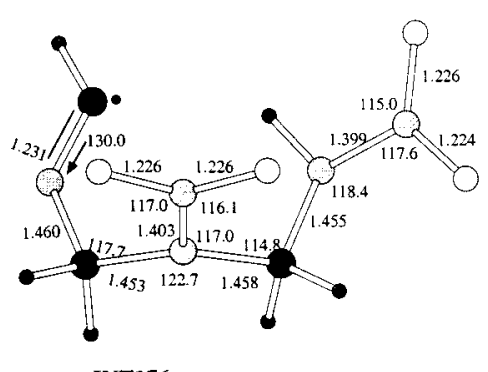

INT176

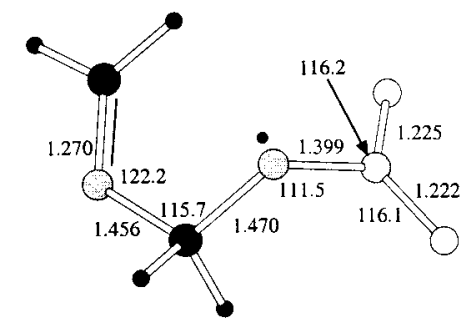

INT102

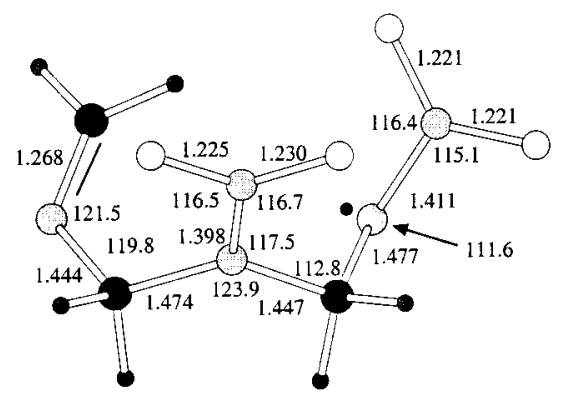

RDR-o
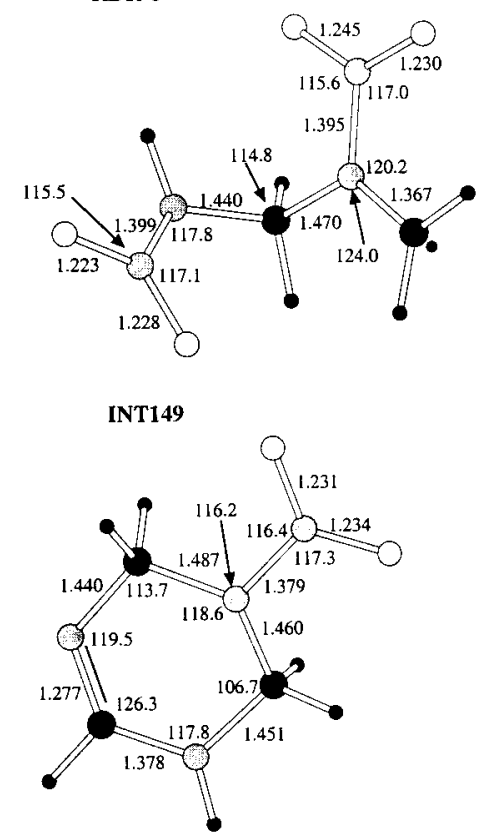

INT130
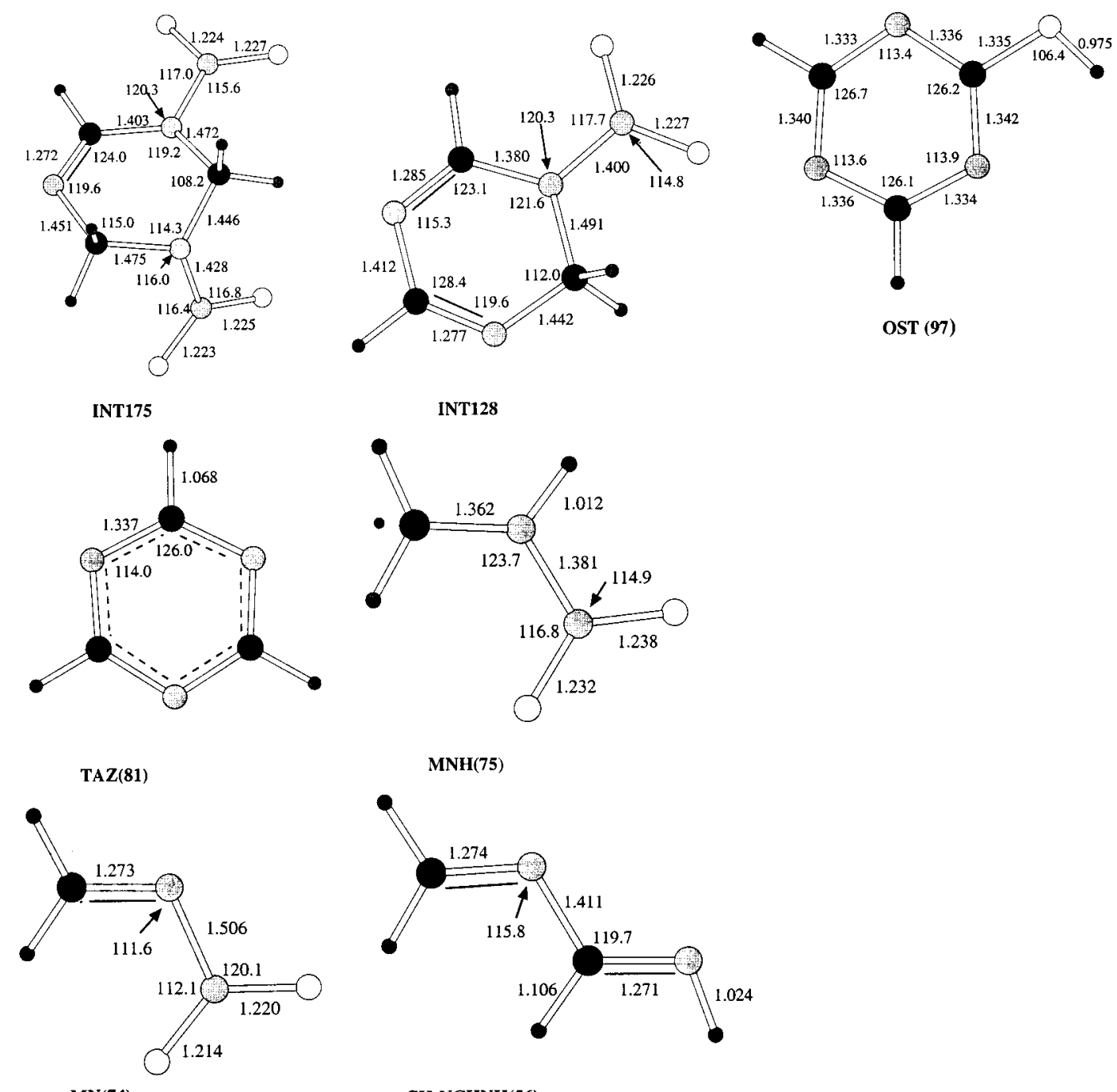

MN(74)

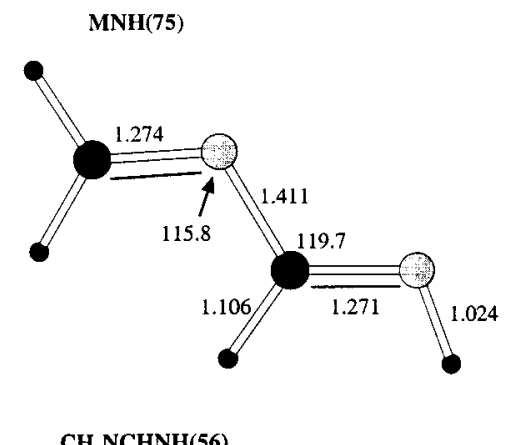

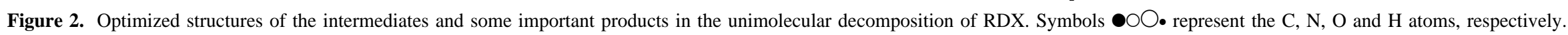




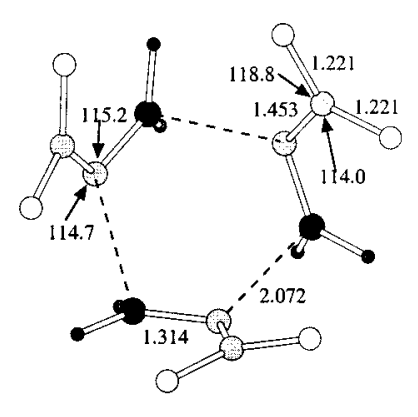

TS1

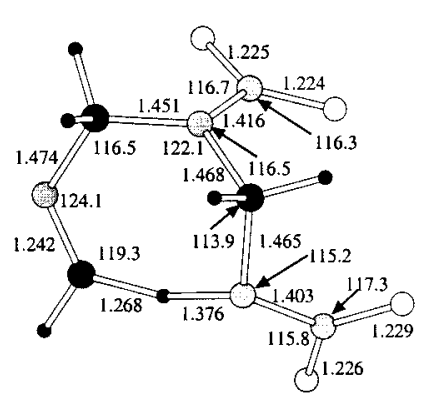

TS3

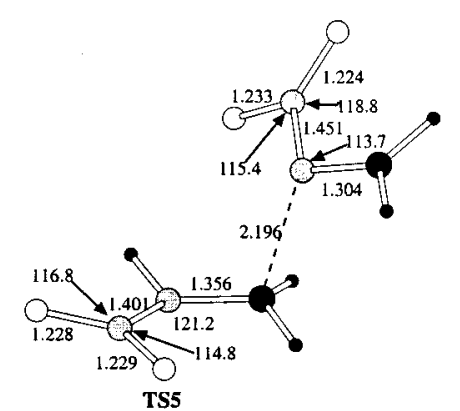

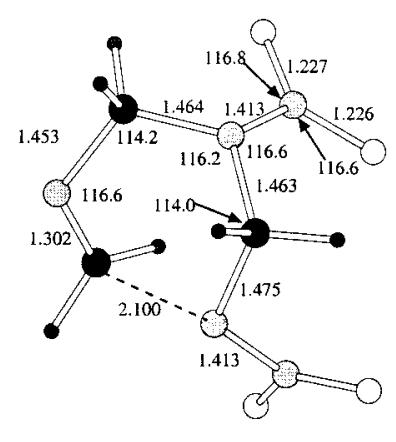

TS2

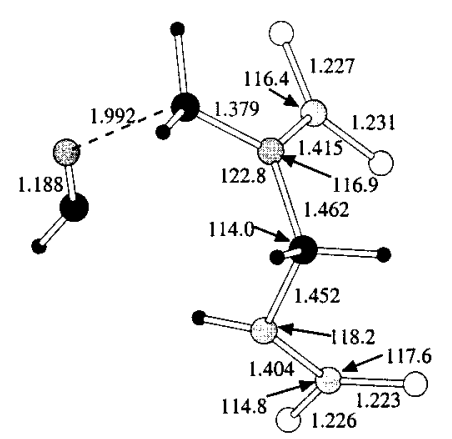

TS4

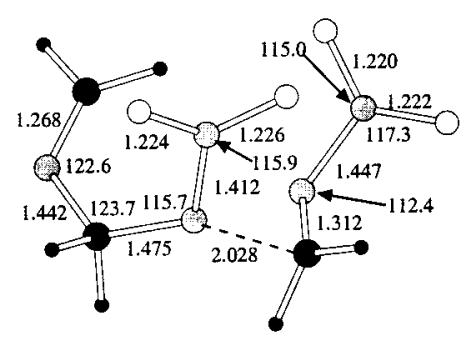

TS6

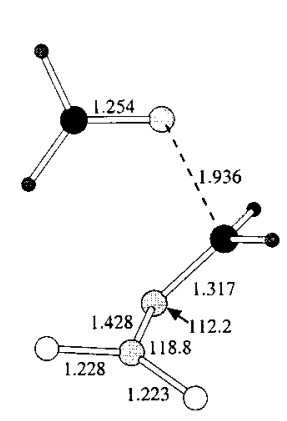

TS7

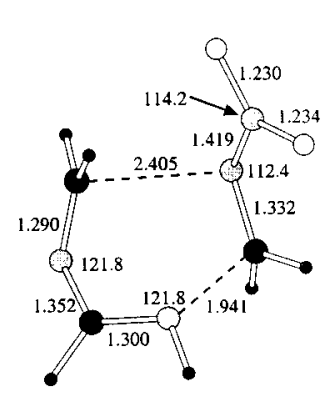

TS9

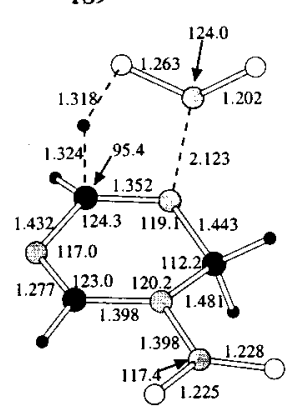

TS11
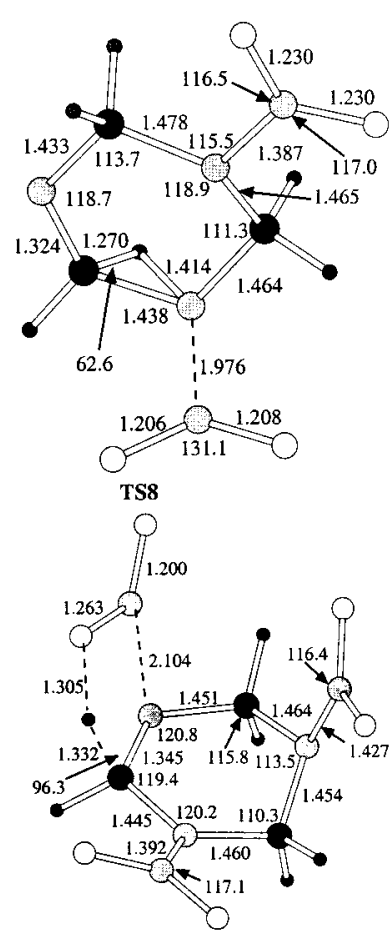

TS10

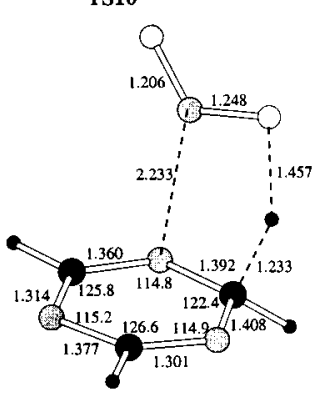

TS12

Figure 3. Optimized structure of the transition states in the unimolecular decomposition of RDX. Symbols $\bullet \circ \bullet$ stands for C, N, O, and $\mathrm{H}$ atoms, respectively.
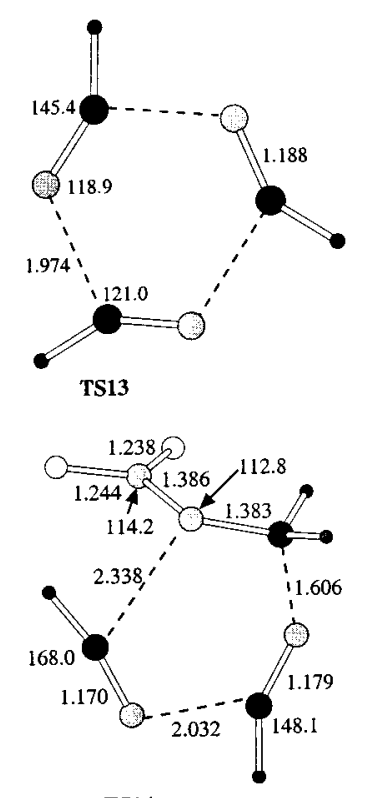

TS14 
TABLE 1: Calculated Frequencies of the Transition States at the B3LYP/6-31G(d) Level of Theory ${ }^{a}$

\begin{tabular}{|c|c|c|c|c|c|c|c|c|c|c|c|c|c|c|}
\hline \multicolumn{15}{|c|}{ TS1 } \\
\hline-397 & 23 & 32 & 32 & 74 & 74 & 77 & 132 & 133 & 176 & 190 & 190 & 380 & 381 & 404 \\
\hline 408 & 409 & 454 & 587 & 587 & 595 & 655 & 657 & 657 & 736 & 740 & 741 & 840 & 840 & 867 \\
\hline 935 & 937 & 940 & 1067 & 1114 & 1114 & 1193 & 1205 & 1205 & 1311 & 1311 & 1335 & 1363 & 1389 & 1389 \\
\hline 1523 & 1543 & 1544 & 1670 & 1694 & 1694 & 3163 & 3163 & 3164 & 3265 & 3265 & 3266 & & & \\
\hline $\begin{array}{r}-567 \\
637 \\
1273 \\
3107\end{array}$ & $\begin{array}{r}54 \\
708 \\
1295 \\
3174\end{array}$ & $\begin{array}{r}67 \\
761 \\
1330 \\
3212\end{array}$ & $\begin{array}{r}81 \\
762 \\
1348\end{array}$ & $\begin{array}{r}99 \\
811 \\
1358\end{array}$ & $\begin{array}{r}179 \\
858 \\
1385\end{array}$ & $\begin{array}{r}249 \\
873 \\
1431\end{array}$ & $\begin{array}{r}\text { TS2 } \\
276 \\
934 \\
1472\end{array}$ & $\begin{array}{r}321 \\
946 \\
1487\end{array}$ & $\begin{array}{r}354 \\
971 \\
1558\end{array}$ & $\begin{array}{r}411 \\
999 \\
1623\end{array}$ & $\begin{array}{r}450 \\
1039 \\
1658\end{array}$ & $\begin{array}{r}511 \\
1114 \\
3052\end{array}$ & $\begin{array}{r}547 \\
1168 \\
3065\end{array}$ & $\begin{array}{r}599 \\
1187 \\
3094\end{array}$ \\
\hline $\begin{array}{r}-1392 \\
614 \\
1226 \\
3116\end{array}$ & $\begin{array}{r}56 \\
656 \\
1304 \\
3174\end{array}$ & $\begin{array}{r}66 \\
698 \\
1323 \\
3184\end{array}$ & $\begin{array}{r}79 \\
749 \\
1344\end{array}$ & $\begin{array}{r}106 \\
765 \\
1347\end{array}$ & $\begin{array}{r}123 \\
779 \\
1380\end{array}$ & $\begin{array}{r}244 \\
856 \\
1410\end{array}$ & $\begin{array}{r}\text { TS3 } \\
278 \\
881 \\
1467\end{array}$ & $\begin{array}{r}326 \\
899 \\
1488\end{array}$ & $\begin{array}{r}355 \\
925 \\
1538\end{array}$ & $\begin{array}{r}420 \\
985 \\
1635\end{array}$ & $\begin{array}{r}429 \\
1049 \\
1673\end{array}$ & $\begin{array}{r}491 \\
1126 \\
1813\end{array}$ & $\begin{array}{r}529 \\
1147 \\
3085\end{array}$ & $\begin{array}{r}599 \\
1210 \\
3092\end{array}$ \\
\hline $\begin{array}{r}-604 \\
594 \\
1230 \\
3312\end{array}$ & $\begin{array}{r}49 \\
602 \\
1280 \\
3325\end{array}$ & $\begin{array}{r}58 \\
615 \\
1315 \\
3476\end{array}$ & $\begin{array}{r}71 \\
629 \\
1359\end{array}$ & $\begin{array}{r}90 \\
676 \\
1366\end{array}$ & $\begin{array}{r}103 \\
698 \\
1413\end{array}$ & $\begin{array}{r}117 \\
754 \\
1452\end{array}$ & $\begin{array}{r}\text { TS4 } \\
159 \\
770 \\
1465\end{array}$ & $\begin{array}{r}220 \\
785 \\
1502\end{array}$ & $\begin{array}{r}254 \\
859 \\
1660\end{array}$ & $\begin{array}{r}333 \\
925 \\
1706\end{array}$ & $\begin{array}{r}414 \\
971 \\
1914\end{array}$ & $\begin{array}{r}429 \\
1008 \\
3097\end{array}$ & $\begin{array}{r}445 \\
1062 \\
3176\end{array}$ & $\begin{array}{r}542 \\
1124 \\
3194\end{array}$ \\
\hline $\begin{array}{r}-348 \\
680 \\
1480\end{array}$ & $\begin{array}{r}43 \\
711 \\
1525\end{array}$ & $\begin{array}{r}46 \\
768 \\
1644\end{array}$ & $\begin{array}{r}67 \\
777 \\
1691\end{array}$ & $\begin{array}{r}74 \\
851 \\
3143\end{array}$ & $\begin{array}{r}174 \\
858 \\
3207\end{array}$ & $\begin{array}{r}250 \\
890 \\
3255\end{array}$ & $\begin{array}{r}\text { TS5 } \\
290 \\
930 \\
3331\end{array}$ & $\begin{array}{r}358 \\
1133 \\
3568\end{array}$ & $\begin{array}{r}387 \\
1160\end{array}$ & $\begin{array}{r}526 \\
1244\end{array}$ & $\begin{array}{r}551 \\
1288\end{array}$ & $\begin{array}{r}579 \\
1333\end{array}$ & $\begin{array}{r}608 \\
1369\end{array}$ & $\begin{array}{r}641 \\
1427\end{array}$ \\
\hline $\begin{array}{r}-454 \\
568 \\
1212 \\
3141\end{array}$ & $\begin{array}{r}51 \\
600 \\
1295 \\
3175\end{array}$ & $\begin{array}{r}63 \\
640 \\
1306 \\
3242\end{array}$ & $\begin{array}{r}74 \\
668 \\
1342\end{array}$ & $\begin{array}{r}91 \\
773 \\
1367\end{array}$ & $\begin{array}{r}108 \\
776 \\
1376\end{array}$ & $\begin{array}{r}129 \\
844 \\
1442\end{array}$ & $\begin{array}{r}\text { TS6 } \\
174 \\
855 \\
1525\end{array}$ & $\begin{array}{r}190 \\
911 \\
1537\end{array}$ & $\begin{array}{r}233 \\
931 \\
1618\end{array}$ & $\begin{array}{r}323 \\
988 \\
1659\end{array}$ & $\begin{array}{r}341 \\
1026 \\
1754\end{array}$ & $\begin{array}{r}384 \\
1082 \\
3036\end{array}$ & $\begin{array}{r}498 \\
1096 \\
3090\end{array}$ & $\begin{array}{r}556 \\
1190 \\
3095\end{array}$ \\
\hline $\begin{array}{r}-475 \\
1084\end{array}$ & $\begin{array}{r}55 \\
1195\end{array}$ & $\begin{array}{r}125 \\
1344\end{array}$ & $\begin{array}{r}149 \\
1360\end{array}$ & $\begin{array}{r}270 \\
1417\end{array}$ & $\begin{array}{r}341 \\
1541\end{array}$ & $\begin{array}{r}419 \\
1639\end{array}$ & $\begin{array}{r}\text { TS7 } \\
505 \\
1718\end{array}$ & $\begin{array}{r}584 \\
3033\end{array}$ & $\begin{array}{r}624 \\
3112\end{array}$ & $\begin{array}{r}793 \\
3119\end{array}$ & $\begin{array}{r}870 \\
3228\end{array}$ & 977 & 1040 & 1049 \\
\hline $\begin{array}{r}-2111 \\
596 \\
1250 \\
3095\end{array}$ & $\begin{array}{r}40 \\
679 \\
1281 \\
3159\end{array}$ & $\begin{array}{r}58 \\
765 \\
1325 \\
3167\end{array}$ & $\begin{array}{r}66 \\
774 \\
1352\end{array}$ & $\begin{array}{r}115 \\
817 \\
1370\end{array}$ & $\begin{array}{r}157 \\
846 \\
1386\end{array}$ & $\begin{array}{r}190 \\
893 \\
1426\end{array}$ & $\begin{array}{r}\text { TS8 } \\
238 \\
942 \\
1476\end{array}$ & $\begin{array}{r}257 \\
993 \\
1507\end{array}$ & $\begin{array}{r}260 \\
999 \\
1517\end{array}$ & $\begin{array}{r}322 \\
1010 \\
1642\end{array}$ & $\begin{array}{r}400 \\
1128 \\
1718\end{array}$ & $\begin{array}{r}448 \\
1150 \\
1934\end{array}$ & $\begin{array}{r}514 \\
1197 \\
2975\end{array}$ & $\begin{array}{r}535 \\
1216 \\
3026\end{array}$ \\
\hline $\begin{array}{r}-364 \\
835 \\
1622\end{array}$ & $\begin{array}{r}41 \\
884 \\
1640\end{array}$ & $\begin{array}{r}74 \\
1000 \\
1676\end{array}$ & $\begin{array}{r}112 \\
1013 \\
3103\end{array}$ & $\begin{array}{r}152 \\
1072 \\
3117\end{array}$ & $\begin{array}{r}221 \\
1078 \\
3137\end{array}$ & $\begin{array}{r}297 \\
1169 \\
3228\end{array}$ & $\begin{array}{r}\text { TS9 } \\
372 \\
1185 \\
3231\end{array}$ & $\begin{array}{r}414 \\
1210 \\
3495\end{array}$ & $\begin{array}{r}466 \\
1263\end{array}$ & $\begin{array}{r}614 \\
1306\end{array}$ & $\begin{array}{r}650 \\
1349\end{array}$ & $\begin{array}{r}664 \\
1416\end{array}$ & $\begin{array}{r}760 \\
1500\end{array}$ & $\begin{array}{r}785 \\
1503\end{array}$ \\
\hline $\begin{array}{r}-1509 \\
458 \\
966 \\
1446\end{array}$ & $\begin{array}{r}31 \\
502 \\
1011 \\
1492\end{array}$ & $\begin{array}{r}50 \\
550 \\
1036 \\
1501\end{array}$ & $\begin{array}{r}63 \\
596 \\
1178 \\
1562\end{array}$ & $\begin{array}{r}85 \\
601 \\
1203 \\
1656\end{array}$ & $\begin{array}{r}109 \\
633 \\
1265 \\
1682\end{array}$ & $\begin{array}{r}132 \\
681 \\
1279 \\
1776\end{array}$ & $\begin{array}{r}\text { TS10 } \\
157 \\
736 \\
1287 \\
3039\end{array}$ & $\begin{array}{r}209 \\
747 \\
1303 \\
3071\end{array}$ & $\begin{array}{r}249 \\
795 \\
1314 \\
3087\end{array}$ & $\begin{array}{r}298 \\
806 \\
1328 \\
3175\end{array}$ & $\begin{array}{r}347 \\
859 \\
1346 \\
3205\end{array}$ & $\begin{array}{r}376 \\
905 \\
1373\end{array}$ & $\begin{array}{r}406 \\
931 \\
1379\end{array}$ & $\begin{array}{r}443 \\
939 \\
1411\end{array}$ \\
\hline $\begin{array}{r}-1485 \\
655 \\
1310\end{array}$ & $\begin{array}{r}40 \\
679 \\
1317\end{array}$ & $\begin{array}{r}80 \\
737 \\
1350\end{array}$ & $\begin{array}{r}99 \\
803 \\
1373\end{array}$ & $\begin{array}{r}144 \\
827 \\
1412\end{array}$ & $\begin{array}{r}178 \\
904 \\
1442\end{array}$ & $\begin{array}{r}213 \\
932 \\
1511\end{array}$ & $\begin{array}{r}\text { TS11 } \\
253 \\
966 \\
1572\end{array}$ & $\begin{array}{r}293 \\
994 \\
1664\end{array}$ & $\begin{array}{r}370 \\
1039 \\
1689\end{array}$ & $\begin{array}{r}432 \\
1043 \\
1769\end{array}$ & $\begin{array}{r}481 \\
1135 \\
3016\end{array}$ & $\begin{array}{r}495 \\
1243 \\
3093\end{array}$ & $\begin{array}{r}554 \\
1256 \\
3134\end{array}$ & $\begin{array}{r}610 \\
1271 \\
3225\end{array}$ \\
\hline $\begin{array}{r}-923 \\
1013 \\
3054\end{array}$ & $\begin{array}{r}80 \\
1024 \\
3179\end{array}$ & $\begin{array}{r}119 \\
1079 \\
3182\end{array}$ & $\begin{array}{r}193 \\
1101\end{array}$ & $\begin{array}{r}226 \\
1113\end{array}$ & $\begin{array}{r}297 \\
1249\end{array}$ & $\begin{array}{r}397 \\
1292\end{array}$ & $\begin{array}{r}\text { TS12 } \\
443 \\
1348\end{array}$ & $\begin{array}{r}539 \\
1372\end{array}$ & $\begin{array}{r}651 \\
1413\end{array}$ & $\begin{array}{r}670 \\
1418\end{array}$ & $\begin{array}{r}774 \\
1474\end{array}$ & $\begin{array}{r}797 \\
1593\end{array}$ & $\begin{array}{r}924 \\
1686\end{array}$ & $\begin{array}{r}955 \\
1726\end{array}$ \\
\hline $\begin{array}{r}-608 \\
1882\end{array}$ & $\begin{array}{r}143 \\
2036\end{array}$ & $\begin{array}{r}144 \\
2036\end{array}$ & $\begin{array}{r}266 \\
3389\end{array}$ & $\begin{array}{r}266 \\
3390\end{array}$ & $\begin{array}{r}314 \\
3391\end{array}$ & 504 & $\begin{array}{r}\text { TS13 } \\
505\end{array}$ & 568 & 777 & 806 & 806 & 967 & 1040 & 1042 \\
\hline $\begin{array}{r}-384 \\
773\end{array}$ & $\begin{array}{r}53 \\
789\end{array}$ & $\begin{array}{r}70 \\
843\end{array}$ & $\begin{array}{l}140 \\
914\end{array}$ & $\begin{array}{l}170 \\
947\end{array}$ & $\begin{array}{r}238 \\
1081\end{array}$ & $\begin{array}{r}297 \\
1210\end{array}$ & $\begin{array}{r}\text { TS14 } \\
301 \\
1293\end{array}$ & $\begin{array}{r}379 \\
1309\end{array}$ & $\begin{array}{r}405 \\
1358\end{array}$ & $\begin{array}{r}453 \\
1469\end{array}$ & $\begin{array}{r}577 \\
1580\end{array}$ & $\begin{array}{r}661 \\
2024\end{array}$ & $\begin{array}{r}681 \\
2094\end{array}$ & $\begin{array}{r}753 \\
3098\end{array}$ \\
\hline 3167 & 3334 & 3402 & & & & & & & & & & & & \\
\hline
\end{tabular}

${ }^{a}$ All frequencies are in $\mathrm{cm}^{-1}$, and the negative numbers indicate imaginary frequencies.

TS for interconversion. The optimized structures of the decomposition products are shown in Figure 2, while the various TS are presented in Figure 3. The vibrational frequencies of all the TS are given in Table 1, while the frequencies for the reactants and stable intermediates are given in Table 2.
The potential energy profile of the unimolecular decomposition mechanism is illustrated in Figures 4, 5, and 6. The optimized geometries (XYZ coordinates) of all the reactants, products, intermediates, and TS are included as supplementary information (Tables S1-S11). 
TABLE 2: Calculated Frequencies of the Intermediates and Some Important Products at the B3LYP/6-31G(d) Level of Theory

\begin{tabular}{|c|c|c|c|c|c|c|c|c|c|c|c|}
\hline \multicolumn{12}{|c|}{ RDR } \\
\hline 28 & 57 & 90 & 108 & 122 & 241 & 324 & 341 & 381 & 409 & 442 & 528 \\
\hline 587 & 640 & 650 & 749 & 754 & 779 & 854 & 867 & 876 & 891 & 929 & 954 \\
\hline 970 & 1133 & 1173 & 1206 & 1246 & 1249 & 1300 & 1318 & 1340 & 1342 & 1383 & 1400 \\
\hline 1409 & 1461 & 1463 & 1484 & 1659 & 1679 & 2959 & 2964 & 3074 & 3142 & 3145 & 3215 \\
\hline \multicolumn{12}{|c|}{ RDRO } \\
\hline 51 & 64 & 69 & 78 & 94 & 115 & 182 & 222 & 258 & 345 & 397 & 444 \\
\hline 463 & 506 & 597 & 607 & 675 & 697 & 758 & 780 & 849 & 853 & 942 & 956 \\
\hline 989 & 1000 & 1079 & 1156 & 1212 & 1220 & 1304 & 1332 & 1353 & 1368 & 1387 & 1417 \\
\hline 1462 & 1488 & 1519 & 1634 & 1658 & 1758 & 3070 & 3077 & 3087 & 3131 & 3149 & 3170 \\
\hline \multicolumn{12}{|c|}{ INT176 } \\
\hline 60 & 65 & 73 & 90 & 101 & 157 & 200 & 239 & 268 & 339 & 413 & 420 \\
\hline 496 & 607 & 608 & 632 & 662 & 687 & 757 & 764 & 778 & 867 & 898 & 930 \\
\hline 950 & 1026 & 1100 & 1113 & 1163 & 1226 & 1320 & 1339 & 1352 & 1372 & 1405 & 1418 \\
\hline 1454 & 1471 & 1501 & 1666 & 1700 & 1863 & 3070 & 3085 & 3094 & 3163 & 3166 & 3451 \\
\hline \multicolumn{12}{|c|}{ INT149 } \\
\hline 56 & 77 & 89 & 135 & 210 & 248 & 345 & 374 & 402 & 431 & 447 & 591 \\
\hline 597 & 614 & 663 & 678 & 751 & 779 & 853 & 915 & 948 & 1047 & 1129 & 1239 \\
\hline 1259 & 1316 & 1362 & 1404 & 1414 & 1447 & 1455 & 1484 & 1629 & 1696 & 3133 & 3199 \\
\hline 3233 & 3370 & 3554 & & & & & & & & & \\
\hline \multicolumn{12}{|c|}{ INT102 } \\
\hline 13 & 84 & 165 & 260 & 296 & 455 & 532 & 633 & 672 & 800 & 869 & 877 \\
\hline 989 & 1073 & 1081 & 1224 & 1286 & 1337 & 1372 & 1492 & 1516 & 1624 & 1741 & 3041 \\
\hline 3088 & 3092 & 3175 & & & & & & & & & \\
\hline \multicolumn{12}{|c|}{ INT130 } \\
\hline 82 & 112 & 164 & 208 & 262 & 364 & 386 & 510 & 537 & 603 & 682 & 757 \\
\hline 816 & 939 & 943 & 986 & 1023 & 1028 & 1058 & 1165 & 1196 & 1223 & 1255 & 1286 \\
\hline 1342 & 1372 & 1417 & 1438 & 1514 & 1522 & 1543 & 1634 & 1752 & 2963 & 3021 & 3105 \\
\hline 3146 & 3149 & 3597 & & & & & & & & & \\
\hline \multicolumn{12}{|c|}{ INT175 } \\
\hline 40 & 64 & 79 & 101 & 149 & 250 & 349 & 366 & 407 & 453 & 485 & 549 \\
\hline 582 & 638 & 674 & 736 & 753 & 816 & 859 & 913 & 926 & 937 & 971 & 996 \\
\hline 1064 & 1180 & 1206 & 1275 & 1286 & 1305 & 1342 & 1345 & 1383 & 1388 & 1425 & 1489 \\
\hline 1508 & 1670 & 1682 & 1729 & 3060 & 3074 & 3166 & 3216 & 3220 & & & \\
\hline \multicolumn{12}{|c|}{ INT128 } \\
\hline 64 & 111 & 166 & 259 & 340 & 391 & 547 & 588 & 606 & 697 & 736 & 832 \\
\hline 908 & 936 & 991 & 996 & 1051 & 1084 & 1126 & 1268 & 1273 & 1317 & 1378 & 1408 \\
\hline 1438 & 1528 & 1631 & 1673 & 1716 & 3047 & 3081 & 3179 & 3221 & & & \\
\hline \multicolumn{12}{|c|}{ TAZ } \\
\hline 353 & 353 & 689 & 689 & 755 & 936 & 1009 & 1030 & 1030 & 1151 & 1187 & 1196 \\
\hline 1196 & 1406 & 1444 & 1444 & 1602 & 1602 & 3175 & 3175 & 3181 & & & \\
\hline \multicolumn{12}{|c|}{ MNH } \\
\hline 159 & 269 & 307 & 334 & 576 & 587 & 685 & 686 & 917 & 1143 & 12330 & 1331 \\
\hline 1435 & 1474 & 1671 & 3230 & 3368 & 3599 & & & & & & \\
\hline \multicolumn{12}{|c|}{ MN } \\
\hline $\begin{array}{r}34 \\
1718\end{array}$ & $\begin{array}{r}398 \\
3126\end{array}$ & $\begin{array}{r}523 \\
3236\end{array}$ & 553 & 633 & 828 & 870 & 1080 & 1181 & 1349 & 1424 & 1683 \\
\hline \multicolumn{12}{|c|}{$\mathrm{CH}_{2} \mathrm{NCHNH}$} \\
\hline 123 & 378 & 627 & 633 & 911 & 979 & 1068 & 1087 & 1208 & 1301 & 1437 & 1505 \\
\hline 1700 & 1728 & 2950 & 3004 & 3167 & 3436 & & & & & & \\
\hline
\end{tabular}

Here and in the discussion, the energies are corrected for ZPE. In discussions, where this correction is not made, we will indicate so by adding: (no ZPE).

III.A. Equilibrium Structure of RDX. The various ring conformations of RDX have been studied extensively, with detailed reviews in two recent theoretical papers. ${ }^{22,23}$ Our predictions are quite similar to these earlier calculations and will not be discussed in detail.

The triaxial chair (AAA) with $C_{3 V}$ symmetry is the global minima, which agrees well with the earlier theoretical results ${ }^{22,23}$ and the gas-phase electron diffraction structure. ${ }^{31}$ We find short $(2.22 \AA)(\mathrm{N}-\mathrm{O}$....H-C) $\mathrm{H}$-bond intramolecular contacts that might aid the intramolecular $\mathrm{H}$-transfer required for a $\mathrm{HONO}$ elimination primary dissociation pathway (also pointed out by Harris and Lammertsma ${ }^{22}$ ).

The tri-equatorial chair conformer (EEE) is also found to be a local minima, but it is $4.7 \mathrm{kcal} / \mathrm{mol}$ less stable than AAA.
We find that the AAA-boat conformer of RDX (see Figure 1) is $0.75 \mathrm{kcal} / \mathrm{mol}$ (no ZPE) above the AAA-chair conformation, but it has one small imaginary frequency $\left(24 \mathrm{i} \mathrm{cm}^{-1}\right)$, indicating that AAA-boat is not a stable structure for RDX (at this level of theory). Starting from this structure and minimizing the energy along this negative eigenvalue leads to the non symmetric twist boat conformer. We attempted to locate the TS corresponding to the boat to AAA chair interconversion. However, the TS converged to a higher order saddle point with two imaginary frequencies $\left(134 \mathrm{i}\right.$ and $\left.10 \mathrm{i} \mathrm{cm}^{-1}\right)$. The $10 \mathrm{i} \mathrm{cm}-1$ mode also corresponds to the boat to twist boat interconversion. Thus, the energy barrier for boat to AAA-chair interconversion is $4.3 \mathrm{kcal} / \mathrm{mol}$ (no ZPE correction).

On the basis of RHF/6-31G* calculations, Harris and Lammertsma $^{22}$ reported that the boat form of RDX is a stable minima. Using the B3LYP/6-311G**//B3LYP/6-31G* level they predict that the boat form of RDX is higher than the AAA 


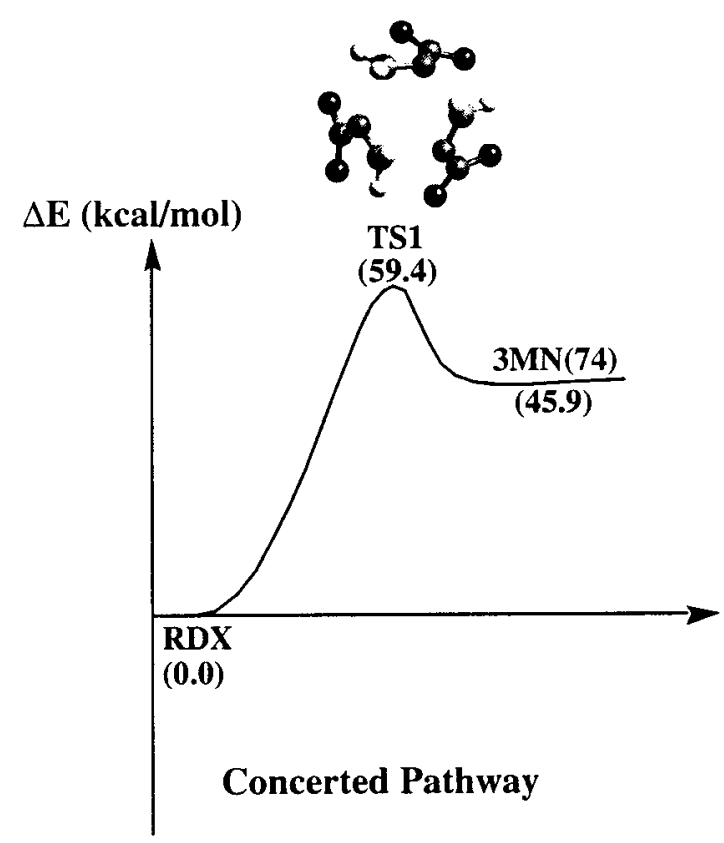

Figure 4. Potential energy profile for the concerted decomposition of RDX.

chair by $0.67 \mathrm{kcal} / \mathrm{mol}$ (no ZPE) which agrees very well with our calculated $\Delta E$ of $0.75 \mathrm{kcal} / \mathrm{mol}$ (no ZPE). The structure of the boat conformer appears to be similar for both calculations.

III.B. Reaction Pathway 1. The Concerted Ring Fission. As mentioned above, the experiments by $\mathrm{ZHL}^{16}$ were interpreted in terms of concerted ring breaking as the primary decomposition path of RDX.

For this process we computed a TS at $59.4 \mathrm{kcal} / \mathrm{mol}$ and a reaction $\Delta H$ of $45.9 \mathrm{kcal} / \mathrm{mol}$, as shown in Figure 4 . The transition structure (denoted TS1) was located by first scanning the reaction path assuming a symmetric structure, followed by optimizing the maximum energy structure along the reaction path. Then starting with this structure we found the closest saddle point (one negative eigenvalue). We did not require TS1 to retain the $C_{3 V}$ symmetry of RDX. At TS1 the breaking $\mathrm{C}-\mathrm{N}$ bonds are $\sim 2.07 \AA$ (1.460 $\AA$ is equilibrium) while all other $\mathrm{C}-\mathrm{N}$ bonds $(\sim 1.31 \AA)$ retain character between the $\mathrm{C}-\mathrm{N}$ single bond $(\sim 1.46 \AA)$ and double bond $(\sim 1.26 \AA)$, as expected. At TS1 all three fragment MN molecules have the nearly planar configuration expected for the free molecule (shown in Figure $3)$.

As discussed in Section I.B, the theoretical analyses by Thompson ${ }^{25}$ indicated that a concerted dissociation cannot be the primary decomposition channel of RDX observed experimentally by ZHL if the barrier is above $40 \mathrm{kcal} / \mathrm{mol}$. Thus, our result that the barrier is $59.4 \mathrm{kcal} / \mathrm{mol}$ suggests that the primary decomposition pathway involves some other process.

Several previous theoretical studies have been carried out on the energetics of the concerted ring opening process. ${ }^{26,27}$ The most complete predictions of the barrier were recently reported by $\mathrm{Wu}$ and Fried. ${ }^{27}$ Based on an extensive series of DFT calculations with a variety of basis sets they recommended the $\mathrm{B}-\mathrm{PW} 91 / \mathrm{cc}-\mathrm{PVDZ}$ level of theory. This leads to $E_{\text {act }}=55.4$ and $\Delta H=44.6 \mathrm{kcal} / \mathrm{mol}$ (including ZPE) as compared to our results of 59.4 and $45.9 \mathrm{kcal} / \mathrm{mol}$ at the B3LYP/6-31G(d) level. (Further, ref 27 quotes $E_{\text {act }}=52.5$ and $\Delta H=41.8 \mathrm{kcal} / \mathrm{mol}$ rather than 55.4 and $44.6 \mathrm{kcal} / \mathrm{mol}$. They inform us (private communication) that there was a misprint in their paper and that they agree with our results.)
III.C. Reaction Path 2. Homolytic $\mathbf{N}-\mathbf{N}$ Bond Fission plus Subsequent Decomposition. III.C.A. $\mathrm{N}-\mathrm{NO}_{2}$ Homolysis to Form RDR. Since homolytic cleavage of single bonds generally do not have appreciable reverse barriers, the first $\mathrm{N}-\mathrm{NO}_{2}$ bond breaking in $\mathrm{RDX}^{21,27}$ has long been considered to be a likely low-energy decomposition channel.

We calculated an $\mathrm{N}-\mathrm{N}$ bond dissociation energy of $39.0 \mathrm{kcal} /$ mol (including ZPE). Since the $\mathrm{N}-\mathrm{N}$ bond energy for RDX is $20 \mathrm{kcal} / \mathrm{mol}$ lower than the concerted mechanism, it is plausible that initiation of the RDX decomposition occurs through the $\mathrm{N}-\mathrm{N}$ bond dissociation.

The calculated $\mathrm{N}-\mathrm{N}$ bond dissociation energy of RDX is slightly higher than the $\mathrm{N}-\mathrm{N}$ bond in $\mathrm{MN}$ (see Figure 2) of $35.9 \mathrm{kcal} / \mathrm{mol}$ (including $\mathrm{ZPE}$ ) at the $\mathrm{G} 2 \mathrm{M}$ level of theory (B3LYP leads to $30.0 \mathrm{kcal} / \mathrm{mol}){ }^{32}$ The estimated $\mathrm{N}-\mathrm{N}$ bond dissociation energy [at the B3LYP/6-311G(d,p)//B3LYP/6-31G(d) level with scaled HF/6-31G(d) ZPE correction] in related nitramines are 46 (dimethylnitramine), 48 (1-nitropiperidine), and 49 (1-nitro-1,3,5-triazine) (where in each case $6 \mathrm{kcal} / \mathrm{mol}$ was added to correct the energy for expected error). ${ }^{22}$

Starting from the pioneering work of Melius and Binkley, ${ }^{21}$ predicted values of the $\mathrm{N}-\mathrm{N}$ bond dissociation energy of RDX have ranged from 35 to $48 \mathrm{kcal} / \mathrm{mol}$. The most recent studies predicted $\mathrm{N}-\mathrm{N}$ bond dissociation energies of 34.2 ( $\mathrm{Wu}$ and Fried $^{27}$ ) and $42.0 \mathrm{kcal} / \mathrm{mol}$ (Harris and Lammertsma ${ }^{22}$ ). The 42.0 $\mathrm{kcal} / \mathrm{mol}$ dissociation energy recommended by Harris and Lammertsma, included a correction of $+6.0 \mathrm{kcal} / \mathrm{mol}$ based on the predicted underestimation of $\mathrm{C}-\mathrm{N}$ bond energies by similar DFT calculations on related small molecules compared to much more accurate $\mathrm{G} 2$ energies.

Although there is some uncertainty in the exact numbers, all reliable calculations indicate that the $\mathrm{N}-\mathrm{N}$ bond cleavage is $\sim 20 \mathrm{kcal} / \mathrm{mol}$ more favorable than the concerted ring fission.

III.C.B. Subsequent Decomposition of RDR. The RDR radical formed after the elimination of first $\mathrm{NO}_{2}$ can undergo further decomposition through two distinct reaction channels as shown in Figure 5. The possibility of other decomposition channels from RDR cannot be ruled out, but we find that the two reaction paths presented here are energetically the most favorable. Melius and Binkley proposed several possible decomposition schemes from RDR, ${ }^{21}$ including these two.

The minimum energy conformation of RDR has the N-radical center between a planar (half chair) and a nonplanar (chair) conformation (see Figure 2). The $\mathrm{N}$ radical in RDR is known ${ }^{22}$ to hyperconjugate with the neighboring $\mathrm{C}-\mathrm{H}$ hydrogens, providing extra stability to the radical and weakening the bond.

III.C.B.1. The Ring Opening Pathway for RDR Decomposition. III.C.B.1a. Formation of RDR-o $+\mathrm{NO}_{2}$. The most commonly considered decomposition pathway is the opening of the RDR ring. ${ }^{21} \mathrm{We}$ also find ring opening of the radical to be the least energy pathway, requiring an additional $26.4 \mathrm{kcal} /$ mol energy. The ring opened structure (RDR-o) is $13.5 \mathrm{kcal} /$ mol less stable than RDR. Two additional possible pathways are concerted ring fission and HONO elimination from RDR. However, our analysis of the product energies confirmed that these two processes cannot compete with the ring opening channel.

The ring opening of RDX preferably occurs through the breaking of the $\mathrm{C}-\mathrm{N}$ bond between the $\mathrm{C}$ atom adjacent to the radical center and its next nitrogen atom. TS2 was generated by first scanning the dissociating $\mathrm{C}-\mathrm{N}$ bond to find a candidate TS. The $\mathrm{C}-\mathrm{N}$ bond was varied from 1.5 to $2.4 \AA$ with an interval of $0.1 \AA$, and each point along the reaction coordinates was then optimized fully (except the reaction coordinate). The 


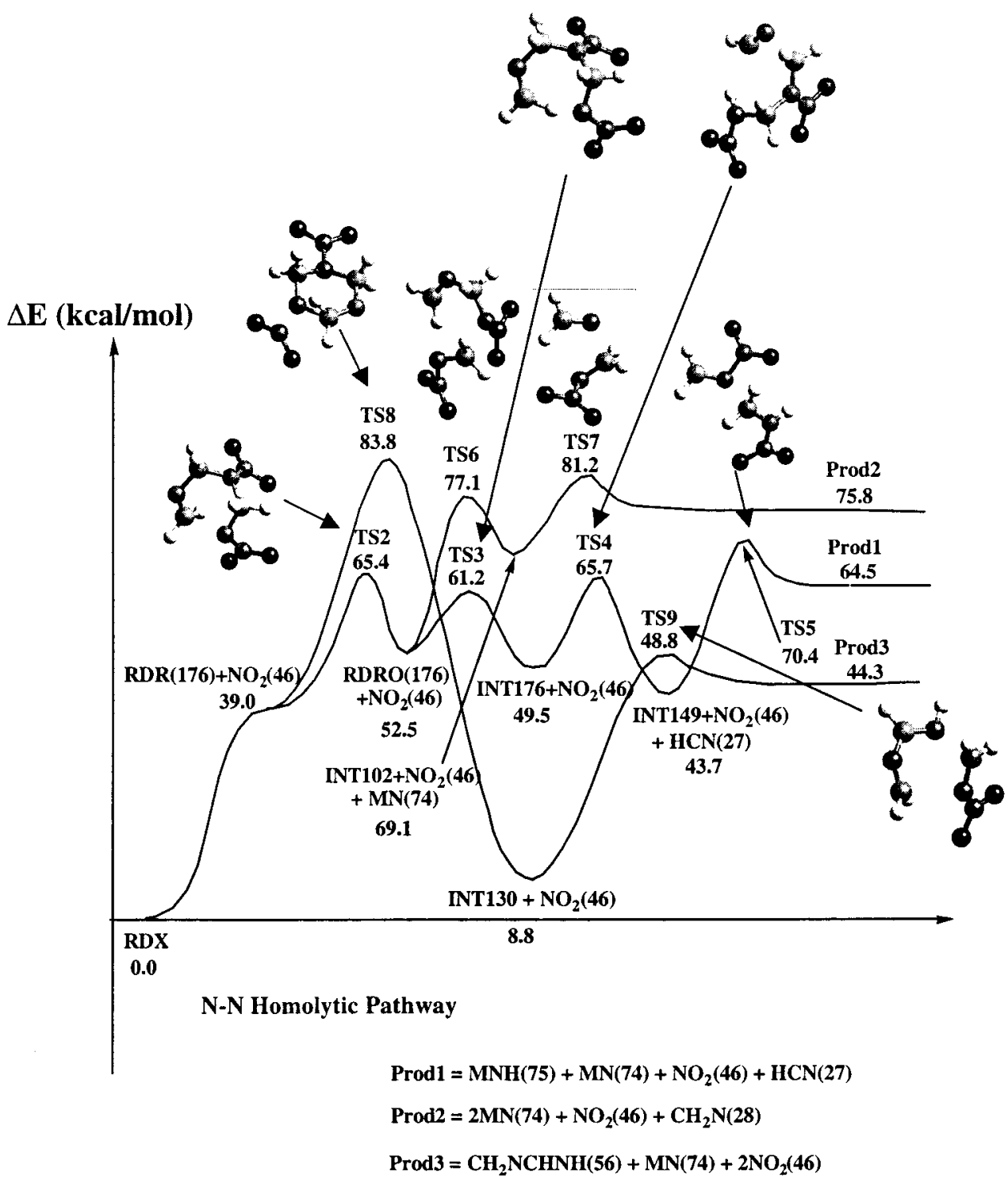

Figure 5. Potential energy profile for the $\mathrm{N}-\mathrm{N}$ bond cleavage and the following decomposition of the radical.

maximum, centered around $2.1 \AA$, was then fully optimized (requiring a single negative curvature) to locate saddle point TS2. To locate the minimum energy structure of RDR-o, we started with the optimized structure at $2.4 \AA$ from the scan and then optimized it fully, which leads to an optimized structure for RDR-o with the nonbonded $\mathrm{C}-\mathrm{N}$ bond at $3.448 \AA$. This confirms that TS2 properly connects RDR and RDR-o.

III.C.B.1b. Decomposition of RDR-o by H Migration Followed by $\mathrm{CN}$ Elimination. A close look at the reaction path from TS2 to RDR-o, reveals that as the $\mathrm{C}-\mathrm{N}$ bond moves further along the reaction path, the $\mathrm{CH}_{2}$ rotates so that the $\mathrm{H}$-atoms approach the breaking $\mathrm{N}$-atom, as shown in Figure 3, leading to a nonbonded contact of $2.798 \AA$ between the $\mathrm{H}$ and $\mathrm{N}$ atom in RDR-o. Indeed, we find that the least energy pathway for decomposition of RDR-o involves H-migration through TS3. The barrier to H-migration is only $8.7 \mathrm{kcal} / \mathrm{mol}$ at TS3, and the isomeric product INT176 is $3.0 \mathrm{kcal} / \mathrm{mol}$ more exothermic than RDR-o.

We searched for a TS for possible elimination of $\mathrm{CH}_{2} \mathrm{~N}$ radical from RDR-o, but these calculations always led to $\mathrm{H}$-migration (because of the favorable $\mathrm{N}-\mathrm{H}$ nonbonded contact in RDR-o).

III.C.B.1c. Decomposition of INT176 (isomer of RDR-o). INT176, which is an isomer of RDR and RDR-o, can eliminate
HCN to form INT149 (a stable radical intermediate) via TS4, which has a barrier of $16.2 \mathrm{kcal} / \mathrm{mol}$ with respect to INT176. The structure of INT149 can be visualized as the result of bonding the $\mathrm{MN}$ molecule to the $\mathrm{CH}_{2} \mathrm{NHNNO}_{2}(\mathrm{MNH})$ radical. The $\mathrm{C}-\mathrm{N}$ bond of the MN fragment in INT149 has partial double bond character $(1.367 \AA)$, whereas the $\mathrm{C}-\mathrm{N}$ bond in the $\mathrm{MNH}$ fragment $(1.440 \AA)$ and the $\mathrm{C}-\mathrm{N}$ bond joining the two moieties $(1.470 \AA)$ have single bond character. Thus, INT149 can easily fission to form $\mathrm{MN}$ and $\mathrm{MNH}$ radical by breaking the common $\mathrm{C}-\mathrm{N}$ bond, the barrier (TS5) of which is $26.7 \mathrm{kcal} / \mathrm{mol}$ above INT149. The product $\mathrm{MNH}$ has $\mathrm{C}-\mathrm{N}$ and $\mathrm{N}-\mathrm{N}$ bond lengths of 1.362 and $1.381 \AA$, respectively, with the radical character centered around the central $\mathrm{N}$-atom.

III.C.B.1d. MN and MNH Decomposition. The structure of $\mathrm{MN}$ is well-known theoretically. ${ }^{33,34}$ The decomposition pathways of $\mathrm{MN}\left(\mathrm{CH}_{2} \mathrm{NNO}_{2}\right)$ are $\mathrm{N}-\mathrm{NO}_{2}$ cleavage, $\mathrm{HCN}+\mathrm{HONO}$ formation, and $\mathrm{H}_{2} \mathrm{CO}+\mathrm{N}_{2} \mathrm{O}$ formation. ZHL reported $\mathrm{HONO}$ formation as the dominant secondary decomposition channel, but recent theoretical results support $\mathrm{N}-\mathrm{N}$ bond breaking as the rate-limiting process. ${ }^{32,34}$

MNH may also decompose in a similar manner either to $\mathrm{CH}_{2}-$ $\mathrm{NH}+\mathrm{NO}_{2}, \mathrm{CH}_{2} \mathrm{~N}+\mathrm{HONO}$, or simple $\mathrm{H}$-elimination to $\mathrm{MN}$ and $\mathrm{H}$. We did not examine these secondary decomposition paths in the present study. 
III.C.B.1e. The Concerted Pathway for RDR-o Decomposition. We searched for a concerted decomposition of RDR-o to two $\mathrm{MN}$ and $\mathrm{CH}_{2} \mathrm{~N}$, but this did not converge to a reliable TS. Rather we found a stepwise mechanism for this process via TS6 and TS7 as shown in Figure 5.

RDR-o can dissociate to INT102 and MN via TS6, leading to a barrier $24.6 \mathrm{kcal} / \mathrm{mol}$ above RDR-o. INT102 can again dissociate to $\mathrm{CH}_{2} \mathrm{~N}$ and $\mathrm{MN}$ by breaking the joining $\mathrm{C}-\mathrm{N}$ bond. The barrier for this process at TS7 is $20 \mathrm{kcal} / \mathrm{mol}$. The breaking $\mathrm{C}-\mathrm{N}$ bond in TS7 is $1.936 \AA$, and both the fragments are close to their respective minimum energy structure.

The final products of the concerted ring opening, $2 \mathrm{CH}_{2} \mathrm{NNO}_{2}$, and $\mathrm{CH}_{2} \mathrm{~N}$ are $36.8 \mathrm{kcal} / \mathrm{mol}$ more endothermic than RDR, as shown in Figure 5. Hence, the associated TS (TS7) is $15.8 \mathrm{kcal} /$ mol higher than TS2. As will be discussed in the next section, the HONO elimination process requires a barrier of $35-40 \mathrm{kcal} /$ mol so that the corresponding TS will also be much higher in energy than TS2.

III.C.B.1f. Elimination of Second $\mathrm{NO}_{2}$ from RDR. The elimination of a second $\mathrm{NO}_{2}$ group from RDR becomes difficult due to formation of a biradical (unpaired spin on each of the two $\mathrm{N}$ radical centers). This high energy conformation can be avoided by $\mathrm{H}$-migration with simultaneous $\mathrm{NO}_{2}$ elimination. This leads to TS8, as shown in Figure 5. As the $\mathrm{N}-\mathrm{NO}_{2}$ distance increases in TS8, the $\mathrm{H}$ atom from the neighboring $\mathrm{CH}_{2}$ group migrates to the $\mathrm{N}$-atom and the adjacent $\mathrm{C}-\mathrm{N}$ bond (between the $\mathrm{N}$ radical in RDR and $\mathrm{H}$ migrating $\mathrm{C}$ ) gains partial double bond character (1.324 $\AA$ ). The breaking $\mathrm{N}-\mathrm{N}$ bond in TS8 is $1.976 \AA$ and the migrating $\mathrm{H}$ atom is $1.414 \AA$ from the $\mathrm{N}$ and $1.270 \AA$ from C. TS8 has a barrier of $44.8 \mathrm{kcal} / \mathrm{mol}$ over RDR and goes to a stable intermediate (INT130).

III.C.B.1g. Concerted Breaking of INT130. INT130 can further decompose concertedly to $\mathrm{CH}_{2} \mathrm{NCHNH}(M=56)$ and $\mathrm{MN}$ via TS9. The breaking $\mathrm{CN}$ bonds in TS9 are 2.405 and $1.941 \AA$, respectively. Both the fragments in TS9 are close to their respective planar structure. The geometry of the $\mathrm{CH}_{2}-$ $\mathrm{NCHNH}$ product is shown in Figure 2.

III.C.C. Summary. The three sets of products formed in the $\mathrm{N}-\mathrm{N}$ homolytic pathway are represented as prod1, prod2, and prod3 in Figure 5. Although the initial dissociation of RDX to form RDR requires less energy than the concerted ring breaking process, the overall endothermicity of three sets of products (prod1, prod2, and prod3) in the former are much higher than that of the products of the concerted ring fission. Thus, an isolated energetic RDX molecule will prefer a concerted ring breaking to form three MN molecules over decomposition of the RDR to form products.

III.D. Reaction Pathway 3. The Concerted HONO Elimination. We find a third pathway, concerted HONO elimination (reaction pathway three), to be more favorable than the concerted and $\mathrm{N}-\mathrm{N}$ homolytic pathways discussed above. This new pathway has not been considered in previous theoretical calculations although $m / e 81$ was experimentally observed by molecular beam ${ }^{16}$ as well as in the pyrolysis experiments. ${ }^{12,13}$

Based on the close nonbonded contact of $\mathrm{H}$ and $\mathrm{NO}_{2}$ in RDX a possibility of $\mathrm{H}$ migration or HONO elimination from RDX was suggested by Harris and Lammertsma. ${ }^{22}$ We searched for a possible $\mathrm{HONO}$ elimination TS from RDX and found that TS10 for the first HONO elimination has a barrier of $39.2 \mathrm{kcal} /$ mol (very close to the calculated $\mathrm{N}-\mathrm{N}$ bond dissociation energy of $39.0 \mathrm{kcal} / \mathrm{mol})$. Such a similarity in energy $(32.7 \mathrm{kcal} / \mathrm{mol}$ for HONO elimination and $35.9 \mathrm{kcal} / \mathrm{mol} \mathrm{N}-\mathrm{N}$ bond dissociation) was also observed in the secondary decomposition of
MN. ${ }^{32}$ This can be attributed to the smaller secondary $\mathrm{C}-\mathrm{H}$ bond strength in nitramines compared to aliphatic or aromatic $\mathrm{C}-\mathrm{H}$ bonds. ${ }^{22}$

For RDX this HONO pathway leads to INT175 and HONO which are $8.5 \mathrm{kcal} / \mathrm{mol}$ exothermic from RDX, as shown in Figure 6. The structure of TS10 is quite similar to the HONO elimination TS observed in $\mathrm{MN}^{32,33}$ or dimethylnitramine ${ }^{35}$ as shown in Figure 3.

III.D.1. Second HONO Elimination. INT175 can eliminate another molecule of HONO via TS11 to form INT128, leading to a TS11 energy $32.0 \mathrm{kcal} / \mathrm{mol}$ above RDX.

III.D.2. Third HONO Elimination. INT128 can subsequently eliminate another HONO molecule via TS12 to form a very stable TAZ (INT81) intermediate. TS12 has an energy of 20.1 $\mathrm{kcal} / \mathrm{mol}$ with respect to RDX. Thus, the decomposition of RDX to $3 \mathrm{HONO}+\mathrm{TAZ}$ is exothermic by $36.4 \mathrm{kcal} / \mathrm{mol}$. Of the various unimolecular decompositions from RDX this is the only one that is exothermic (energy released).

The breaking $\mathrm{N}-\mathrm{N}$ bonds in TS10, TS11, and TS12 are $2.104,2.123$, and 2.233 , and the breaking $\mathrm{C}-\mathrm{H}$ bonds are 1.332, 1.324, and 1.223; whereas the forming $\mathrm{OH}$ bonds are 1.305, 1.318, and 1.457 and the forming $\mathrm{CN} \pi$ bonds are $1.345,1.352$, and $1.392 \AA$, respectively. Due to the formation of a $\mathrm{CN} \pi$ bond via the elimination of HONO in INT175 and INT128, the neighboring $\mathrm{NO}_{2}$ group rearranges from axial to equatorial orientation. INT81 has a longer CN double bond of $1.337 \AA$ compared to INT175(1.272) and INT128(1.277, 1.285), due to the complete delocalization of the $\pi$ cloud which brings a $D_{3 h}$ symmetry to the structure.

III.D.3. TAZ Concerted Decomposition. TAZ is a very stable intermediate. We find that it unzips through a concerted mechanism to three $\mathrm{HCN}$, but with an energy barrier of 88.6 $\mathrm{kcal} / \mathrm{mol}$ (TS13) (located in the same way as TS1). However, considering that TAZ $+3 \mathrm{HONO}$ is $36.4 \mathrm{kcal} / \mathrm{mol}$ more stable than RDX, the overall energy requirement for the decomposition is $52.2 \mathrm{kcal} / \mathrm{mol}$ with respect to RDX.

The final products, $3 \mathrm{HCN}+3 \mathrm{HONO}$ are $14.2 \mathrm{kcal} / \mathrm{mol}$ endothermic from RDX.

TS13 is a symmetric planar TS, with the breaking $\mathrm{CN}$ bonds at $1.974 \AA$ (1.337 $\AA$ equilibrium) while the fragment HCNs are each close $\left(\mathrm{H}-\mathrm{C}-\mathrm{N}\right.$ angles of $\left.145.3^{\circ}\right)$ to the product linear structure.

III.D.4. Concerted Ring Decomposition of INT175 and INT128. Concerted ring breaking of INT175 to two MN and HCN or INT128 to MN and two HCN are conceivably low energy paths for the formation of $\mathrm{MN}$ alternative to the concerted and $\mathrm{N}-\mathrm{N}$ homolysis pathways. Consequently, we searched for possible TS from INT175 and INT128. We did not find a reliable TS from INT175. However, TS14 is a genuine TS for concerted breaking of INT128 to $2 \mathrm{HCN}$ and MN. TS14 has an energy of $51.7 \mathrm{kcal} / \mathrm{mol}$ above RDX. Thus, this could be considered as an additional low energy path for the formation of $\mathrm{MN}$.

III.D.5. Possible Formation of OST. OST (m/e = 97) was observed as one of the major unimolecular decomposition products of RDX in most of the condensed phase decomposition experiments. ${ }^{2,12}$ However, the mass fragment 97 was not detected by $\mathrm{ZHL}$ in their IRMPD study of isolated RDX molecule. ${ }^{16}$ Behrens and Bulusu ${ }^{2}$ proposed an intramolecular rearrangement mechanism for the formation of OST which involved elimination of an HNO and two HONO molecules from RDX. Since the intermediate formed after elimination of two HONO molecules (INT128) is $13.0 \mathrm{kcal} / \mathrm{mol}$ more stable than RDX in the HONO elimination pathway, we believe that 


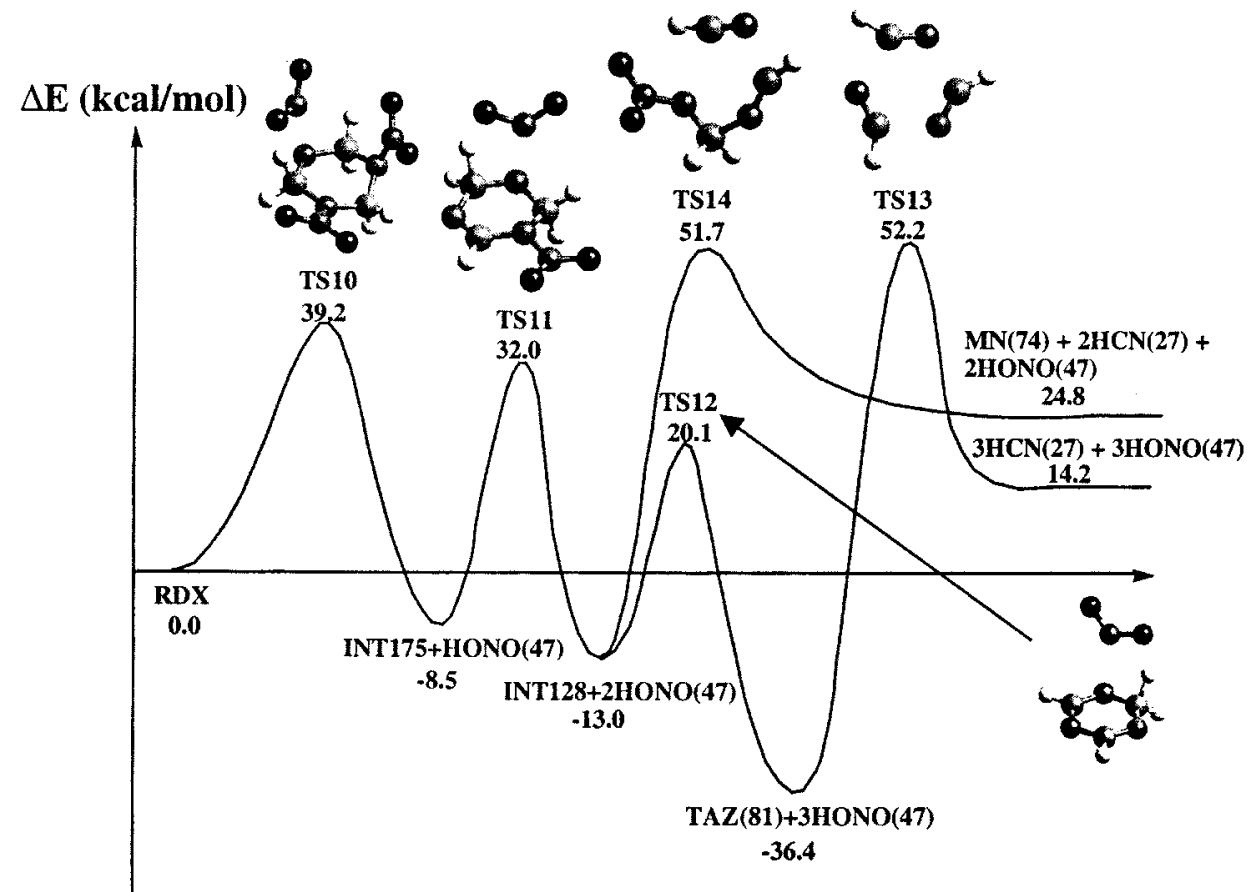

HONO-Elimination Pathway

Figure 6. Potential energy profile of the concerted HONO elimination from RDX and the following decompositions.

the formation of OST will more preferably occur from INT128 via the elimination of an HNO molecule.

Behrens and Bulusu proposed that the mechanism of forming OST from INT128 involves migration of one of the $\mathrm{CH}_{2}$ hydrogens to the oxygen atom of the neighboring $\mathrm{NO}_{2}$ group as a first step. To test this possibility we performed a scan of the nonbonded H...O distance from INT128. As the $\mathrm{H}$ atom approaches the $\mathrm{O}$ atom of the $\mathrm{NO}_{2}$ group, the neighboring $\mathrm{N}-\mathrm{N}$ bond becomes larger and formation of a $\mathrm{C}-\mathrm{N} \pi$ bond occurs which finally lead to the formation of TAZ + HONO. The proposed intermediate after $\mathrm{H}$-migration is also found to be unstable, leading to the decomposition to TAZ + HONO when optimized fully. Since the initial H-migration preferably leads to the elimination of $\mathrm{HONO}$, formation of OST cannot be explained by this mechanism.

On the other hand, our calculations do show OST + HNO $+2 \mathrm{HONO}$ to be energetically $6.9 \mathrm{kcal} / \mathrm{mol}$ more stable than $\mathrm{TAZ}+3 \mathrm{HONO}$. Thus we cannot deny that there might be some other unexplored mechanism for the formation of OST, perhaps a nitro-nitrite rearrangement in INT128 followed by the elimination of $\mathrm{HNO}$ molecule.

\section{Discussion}

Comparison of Experimental Mass Spectra. Our calculations indicate that HONO elimination is the most favorable channel for RDX decomposition and it is the only channel leading to exothermic unimolecular decomposition. Beside establishing the minimum energy pathways for RDX decomposition, a complete mechanism must account for all mass fragments detected in TOFVS (ZHL) ${ }^{16}$ and the laser pyrolysis studies. ${ }^{12}$

We find that the threshold for unimolecular decomposition of RDX is $40 \mathrm{kcal} / \mathrm{mol}$ which forms $\mathrm{RDR}(176)+\mathrm{NO}_{2}(46)$ by $\mathrm{N}-\mathrm{N}$ homolytic and $\mathrm{TAZ}(81)+3 \mathrm{HONO}(47)$ by $\mathrm{HONO}$ elimination.
With a total energy of $\sim 52 \mathrm{kcal}$ more, we expect to see decomposition of TAZ to form $3 \mathrm{HCN}(27)$ plus decomposition of INT128 to form $\mathrm{MN}(74)$ and $2 \mathrm{HCN}(27)$. Furthermore, at an energy of $\sim 50 \mathrm{kcal} / \mathrm{mol}$, we expect decomposition of $\mathrm{HONO}$ to $\mathrm{OH}(17)$ and $\mathrm{NO}(30)$.

With a total energy of $\sim 60 \mathrm{kcal} / \mathrm{mol}$ we expect opening of the concerted pathway to form $3 \mathrm{MN}(74)$.

With $\sim 70 \mathrm{kcal} / \mathrm{mol}$ we expect RDR decomposition to form $\mathrm{MNH}(75), \mathrm{MN}(74)$, and $\mathrm{HCN}(27)$ to become possible.

Finally, with $\sim 80 \mathrm{kcal} / \mathrm{mol}$ or above, RDR decomposition through another two channels (see Figure 5) opens up and it becomes possible to form $\mathrm{MN}(74)$ and $\mathrm{CH}_{2} \mathrm{~N}(28)$ via decomposition of INT102, and to form $\mathrm{CH}_{2} \mathrm{NCHNH}(56), \mathrm{MN}(74)$, and $\mathrm{NO}_{2}(46)$ via INT130.

This leads to the schematic diagram for mass fragments from decomposing RDX at different internal energy as shown in Figure 7. Given the total internal energy of $80 \mathrm{kcal} / \mathrm{mol}$ for RDX molecule in the ZHL laser studies, all the three above channels might be energetically accessible. The major mass fragments detected by ZHL are 120, 102, 81, 74, 56, 46, 44, 42, 26-30, and $12-17$. At around $40 \mathrm{kcal} / \mathrm{mol}$ energy the only mass fragments which could be found are 176(INT176), 81(INT81), $47(\mathrm{HONO})$, and $46\left(\mathrm{NO}_{2}\right) .176$ is a result of $\mathrm{N}-\mathrm{N}$ bond homolytic cleavage and was evident in most of the experiments. ${ }^{16-20 .}$ The mass 81 , which is a major decomposition product found at this energy, was observed as a strong peak by $\mathrm{ZHL}^{16}$ and also by others. ${ }^{12,13}$ On the basis of their translational energy distributions, ZHL considered mass 81 as a faster decomposition component of mass 176 . Our calculation clearly indicates that this is not the case, mass 81 could be formed directly through consecutive HONO elimination (pathway 3). INT175 and INT128 are short-lived intermediates, and hence, these mass fragments are not likely to be observed experimentally.

There was no evidence of $m / e$ 47(HONO) in the IRMPD study of ZHL because it undergoes further decomposition to 


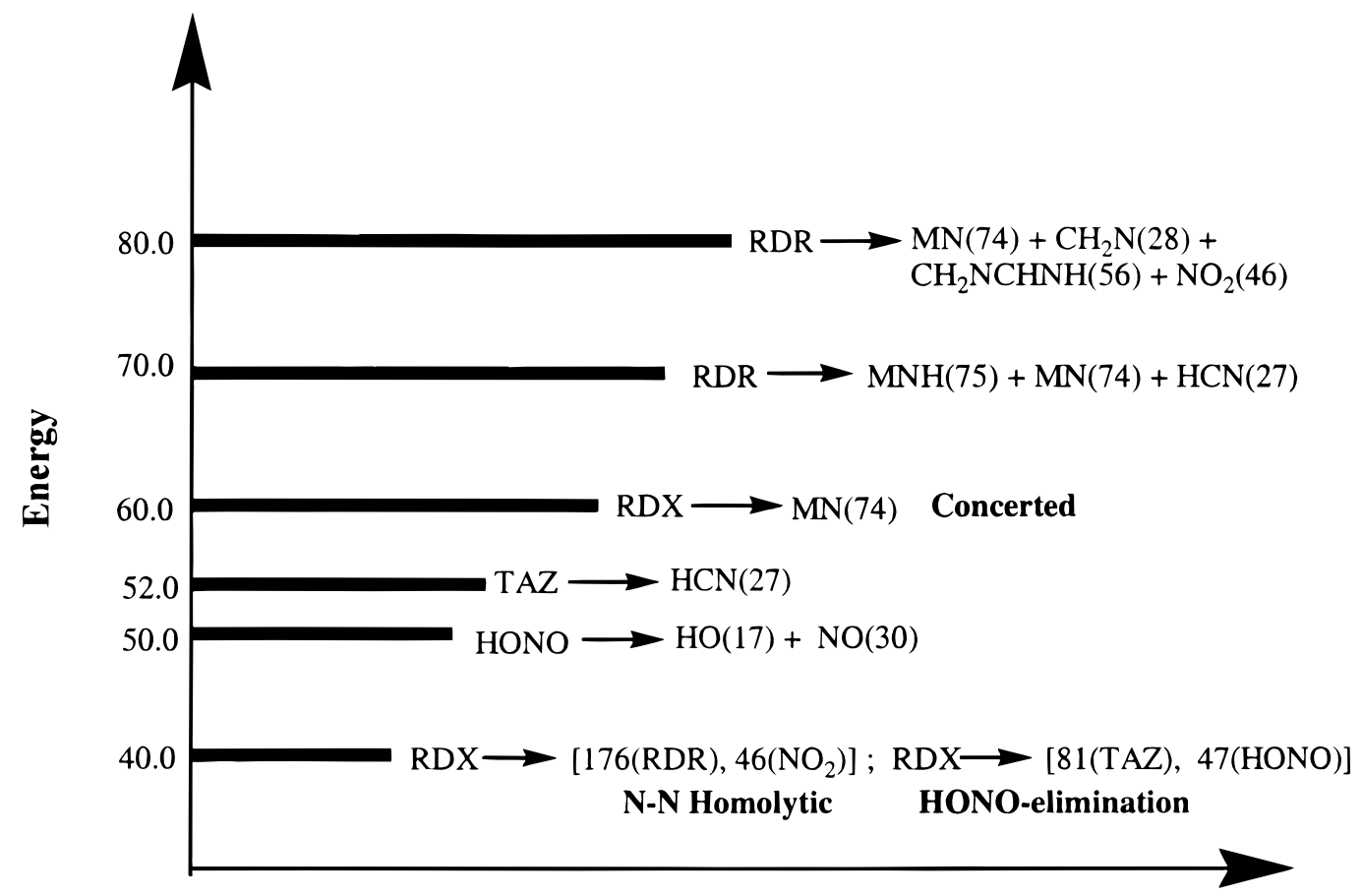

Mass Fragments

Figure 7. Schematic diagram of the decomposing mass fragments at different internal energy.

$\mathrm{OH}(17)$ and $\mathrm{NO}(30)$ with a barrier of $49.6 \mathrm{kcal} / \mathrm{mol}$ (calculated). This is consistent with a recent pyrolysis study of Lee et al., ${ }^{12}$ who observed $m / e 47$ as a parent mass peak (with $m / e 17$ observed experimentally mainly due to decomposition of HONO). ${ }^{16,17}$ However, in ref 16 , ZHL observed only a slower component of $\mathrm{OH}$ generated from the decomposition of secondary $\mathrm{HONO}$ obtained from $\mathrm{CH}_{2} \mathrm{NNO}_{2}(\mathrm{MN})$ decomposition.

At around $52 \mathrm{kcal} / \mathrm{mol}$ energy we expect $27(\mathrm{HCN})$ to be found while at $60 \mathrm{kcal} / \mathrm{mol}$ we expect $74(\mathrm{MN})$ to be formed in addition to the lower energy products. Indeed, 74 was observed as a major mass fragment experimentally. ${ }^{16}$ With $\sim 52 \mathrm{kcal} /$ mol we find $m / e 74$ can be formed through the HONO elimination channel. The faster component of 74 observed by ZHL was considered to be the result of the decomposition of parent RDX molecule through concerted ring breaking. When the internal energy of the molecule is around $60 \mathrm{kcal} / \mathrm{mol}$, reaction path 1 is accessible, which justifies the fast component of 74 found experimentally.

At an energy of $70-80 \mathrm{kcal} / \mathrm{mol}$ the RDR decomposition from $\mathrm{N}-\mathrm{N}$ homolysis pathway is also accessible which could form the mass fragments $75(\mathrm{MNH}), 74(\mathrm{MN}), 27(\mathrm{HCN})$, and 102(INT102). However, RDR-o, INT176, and INT149 are not very stable intermediates and might not be observed experimentally.

We calculate that at 81 to $84 \mathrm{kcal} / \mathrm{mol}$ the decomposition of INT102 and INT130 (see Figure 5) would lead to mass fragments $74(\mathrm{MN}), 28\left(\mathrm{CH}_{2} \mathrm{~N}\right)$, and $56\left(\mathrm{CH}_{2} \mathrm{NCHNH}\right)$. Since the energetics may be off by $\sim 3 \mathrm{kcal} / \mathrm{mol}$ they might also be accessible by the ZHL experiments.

Our calculations account for all mass fragments (including $m / e$ 102, 81, 74, 56, 46, 30, 27, and 17) observed by ZHL in their IRMPD study except for $m / e$ 120, which is not a major product. The possible formula of $\mathrm{CH}_{2} \mathrm{~N}_{3} \mathrm{O}_{4}$ was suggested by ZHL for $m / e$ 120, who considered it as decomposition product of 176(RDR). However, we have not found a rearrangement pathway from RDR leading to formation of 120 . Thus, we cannot understand how 120 could result from a primary decomposition pathway.

The other smaller mass fragments observed experimentally by $\mathrm{ZHL}$, such as $44\left(\mathrm{~N}_{2} \mathrm{O}\right), 29\left(\mathrm{H}_{2} \mathrm{CO}\right)$, etc., result from secondary decomposition of such products as $\mathrm{MN}$. At around $33 \mathrm{kcal} / \mathrm{mol}$ energy $\mathrm{MN}$ can decompose to $47(\mathrm{HONO})$ and $27(\mathrm{HCN})$ whereas $\sim 38 \mathrm{kcal} / \mathrm{mol}$ energy is required to form $44\left(\mathrm{~N}_{2} \mathrm{O}\right)$ and $29\left(\mathrm{H}_{2} \mathrm{CO}\right) .{ }^{32}$ Thus, the (74) $\mathrm{MN}$ resulting from the HONO elimination pathway can undergo further decomposition at an energy $\sim 60 \mathrm{kcal} / \mathrm{mol}$ while, $\sim 80 \mathrm{kcal} / \mathrm{mol}$ or higher energy is required for the secondary decomposition of $\mathrm{MN}$ formed via the other two pathways.

\section{Summary}

Our calculations lead to the following conclusions about RDX decomposition:

(i) Three distinct reaction pathways are identified for the unimolecular decomposition of RDX. The consecutive HONO elimination to form TAZ and 3HONO (with further decomposition of TAZ to $3 \mathrm{HCN}$ ) is identified as the energetically most favorable decomposition pathway. $\mathrm{N}-\mathrm{N}$ bond cleavage to form RDR is also favorable but the associated endothermicity for further decomposition makes this channel less favorable than the other two reaction paths.

(ii) The present scheme successfully accounts for the mass fragments observed in molecular beam experiments except for $m / e 120$.

(iii) The HONO elimination reaction pathway is the most exothermic primary decomposition channel and may account for the energy release observed in the decomposition of RDX. Of course, most of the energy released in the gas-phase comes from secondary reactions.

Acknowledgment. This research was supported by a grant from the DOE-ASCI-ASAP. The MSC facilities activities are also supported by grants from NSF CHE (95-12279), Chevron 
Corp., ARO-MURI, Beckman Institute, Exxon, Owens-Corning, Avery-Dennison, Dow Chemical, 3M, NIH, Asahi Chemical, BP Amoco, and ARO ASSERT.

Supporting Information Available: Tables S1-S11 contain the optimized geometries (XYZ coordinates) of all the reactants, products, intermediates, and TS. This material is available free of charge via the Internet at http://pubs.acs.org.

\section{References and Notes} 11.

(1) Adams, G. F.; Shaw, R. W., Jr. Annu. Rev. Phys. Chem. 1992, 43,

(2) (a) Behrens, R., Jr.; Bulusu, S. J. Phys. Chem. 1992, 96, 8877, 8891. (b) Behrens, R., Jr.; Bulusu, S. J. Phys. Chem. 1991, 95, 5838. (c) Behrens, R., Jr. J. Phys. Chem. 1990, 94, 6706. (d) Behrens, S., Jr. In Chemistry and Physics of Energetic Materials; Bulusu, S. N., Ed.; Kluwer: Dordrecht, 1990; p 347.

(3) Rauch, F. C.; Fanelli, A. J. J. Phys. Chem. 1969, 73, 1604.

(4) Batten, J. J. Aust. J. Chem. 1974, 24, 945.

(5) Cosgrove, J. D.; Owen, A. J. Combust. Flame 1974, 22, 13.

(6) Farber, M.; Srivastave, R. D. Chem. Phys. Lett. 1979, 64, 307.

(7) Doyle, R. J., Jr.; Campana, J. E. J. Phys. Chem. 1985, 89, 4251.

(8) Hoffsommer, J. C.; Glover, D. J. Combust. Flame 1985, 59, 303.

(9) Oxens, F. J.; Sharma, J. Appl. Phys. 1980, 51, 1494.

(10) Bulusu, S.; Weinstein, D. I.; Autera, J. R.; Velicky, R. W. J. Phys. Chem. 1986, 90, 4121.

(11) Brill, T. B.; Gongwer, P. E.; Williams, G. K. J. Phys. Chem. 1994, 98,12242 600.

(12) Lee, Y.; Tang, C.-J.; Litzinger, T. A. Combust. Flame 1999, 117,

(13) Ermolin, N. E.; Zarko, V. E. Combust. Explosion Shock Waves 1997, 33, 251.

(14) Rogers, R. N.; Daub, G. W. Anal. Chem. 1973, 45, 596.
(15) Zuckermann, H.; Greenblatt, G. D.; Haas, Y. J. Phys. Chem. 1987, 91,5159 .

(16) Zhao, X.; Hinsta, E. J.; Lee, Y. T. J. Chem. Phys. 1988, 88, 801.

(17) Capellos, C.; Papagiannakopulous, P.; Liang, Y.-L. Chem. Phys. Lett. 1989, 164, 533

(18) Botcher, T. R.; Wight, C. A. J. Phys. Chem. 1994, 98, 5441; 1993, 97, 9149 .

(19) Pace, M. D. J. Phys. Chem. 1991, 95, 5858.

(20) Choi, M.; Kim, H.; Chung, C. J. Phys. Chem. 1995, 99, 15785.

(21) (a) Melius, C. F.; Binkley, J. S. Symp. (Int.) Combust., [Proc.], $21^{\text {st }}$ 1986, 1953. (b) Melius, C. F. In Chemistry and Physics of Energetic Materials; Bulusu, S. N., Ed.; Kluwer: Dordrecht, 1990; p 21.

(22) Harris, N. J.; Lammertsma, K. J. Am. Chem. Soc.1997, 119, 6583.

(23) Rice, B. M.; Chabalowski, C. F. J. Phys. Chem. A. 1997, 101, 8720.

(24) Sewell, T. D.; Thompson, D. L. J. Phys. Chem. 1991, 95, 6228.

(25) Chambers, C. C.; Thompson, D. L. J. Phys. Chem. 1995, 99, 15881

(26) Habibollahzadeh, D.; Grodzicki, M.; Seminario, J. M.; Politzer, P. J. Phys. Chem. 1991, 95, 7699.

(27) Wu, C. J.; Fried, L. E. J. Phys. Chem. A. 1997, 101, 8675

(28) Becke, A. D. J. Chem. Phys. 1993, 98, 5648, 1372; 1992, 96, 2155; 1992, 97, 9173

(29) Lee, C.; Yang, W.; Parr, R. G. Phys. Rev. B. 1988, 37, 785.

(30) (a) Jaguar 3.5; Schrödinger Inc.: Portland, OR, 1998. (b) Greeley, B. H.; Russo, T. V.; Mainz, D. T.; Friesner, R. A.; Langlois, J-M.; Goddard, W. A., III; Donnelly, R. E.; Ringnalda, M. N. J. Chem. Phys. 1994, 101, 4028.

(31) (a) Shishkov, I. F.; Vilkov, L. V.; Kolonits, M.; Rozsondai, B. Struct. Chem. 1991, 2, 57. (b) Choi, C. S.; Prince, E. Acta Crystallogr. 1972, B28, 2857.

(32) Chakraborty, D.; Lin, M. C. Gas-Phase Chemical Kinetics of [C, $\mathrm{H}, \mathrm{N}, \mathrm{O}]-$ Systems Relevant to the Combustion of Nitramines in Their Early Stages. J. Prop. Power, in press.

(33) Mowrey, R. C.; Page, M.; Adams, G. F.; Lengsfield, B. H., III. J. Chem. Phys. 1990, 93, 1857.

(34) Rice, B. M.; Adams, G. F.; Page, M.; Thompson, D. L. J. Chem. Phys. 1995, 99, 5016.

(35) Harris, N. J.; Lammertsma, K. J. J. Phys. Chem. A. 1997, 101, 1370 